1 In vitro and in vivo evaluation of paclitaxel-induced release of apoptotic 2 biomarker ccCK18 to guide treatment optimization in ovarian cancer

7 Author list

8 Elien De Thaye (1,2), Koen Van de Vijver (2,3), Jo Van Dorpe (2,3), An Vermeulen (1), Jan Van Bocxlaer (1), 9 Pieter Colin $(1,4)$

(1) Laboratory of Medical Biochemistry and Clinical Analysis, Ghent University, Ghent, Belgium

(2) Cancer Research Institute Ghent (CRIG), Ghent, Belgium

(3) Department of Pathology, Ghent University Hospital, Ghent, Belgium

(4) Department of Anesthesiology, University Medical Center Groningen, University of Groningen,

Corresponding author 
33 Background: Cytokeratins hold potential as biomarkers due to their epithelial specificity, abundance and cleavage by caspases during apoptosis. We evaluated paclitaxel-induced circulating caspase-cleaved (cc) cytokeratin 18 (CK18) as potential apoptotic cell-death marker to guide treatment optimization in ovarian cancer.

Methods: Six ovarian cancer cell lines (SK-OV-3, SK-OV-3lucIP1, Caov-3, NIH:OVCAR-3, PA-1 and PM-LGSOC-

01) were exposed in vitro to paclitaxel (PTX, 0 to $1000 \mathrm{nM}$ ) for 24h. Extracellular levels of ccCK18 were measured until 5 days after drug exposure. Cell count and ccCK18 release data were analyzed using a phasenonspecific pharmacodynamic model implemented in NONMEM ${ }^{\circledR}$. PA-1 and SK-OV-3lucIP1 xenografted female SCID/Beige mice received a placebo or single dose of $50 \mathrm{mg} / \mathrm{kg}$ PTX intraperitoneally. Response to PTX was evaluated in vivo using tumor volume and released ccCK18 levels.

Results: In vitro, the correlation between cell count and released ccCK18 levels was present in all cell lines (Spearman's rank correlation coefficient $>0.64$ ). Tumor volume and ccCK18 longitudinal dynamics were markedly different for controls and PTX-treated PA-1 xenografts with changes in ccCK18 release preceding changes in tumor volume. For SK-OV-3lucIP1 xenografts, no differences were found between controls and

47 PTX-treated mice.

Conclusions: An association between PTX-induced ccCK18 release and cell count was demonstrated in vitro. The in vivo study supported the presence of an early-apoptotic peak in ccCK18 levels compared to a later observed effect on tumor volume in PTX-sensitive xenografts. Given the heterogeneous character of ovarian

51 cancer, application and implementation of ccCK18 in a clinical setting to optimize or personalize cancer

52 treatment needs further refinement.

55 ovarian cancer, paclitaxel, caspase-cleaved cytokeratin 18, apoptosis, treatment optimization 
II. Background

57 As a member of the intermediate filament protein family, cytokeratin 18 (CK18) belongs to the type 1 acidic keratins and is expressed in a variety of single-layered and simple epithelia [1]. Besides its role in tumor cell behavior and numerous cellular processes such as apoptosis and cell cycle progression, CK18 is also involved in providing the intracellular scaffolding that structures the cytoplasm, supports normal mitochondrial structures and resists stress applied from the outside of the cell $[2,3]$. Furthermore, this keratin is also used as an epithelial marker in tumor pathology [4].

63 In contrast to its filament partner cytokeratin 8 (CK8), CK18 is a substrate for caspase cleavage during 64 epithelial cell apoptosis [5]. This characteristic makes ccCK18 interesting as a tumor marker that provides information on the extent of ongoing apoptotic tumor cell death. Caspases, a family of aspartate-specific cysteine proteases, contribute frequently to the process of apoptosis and are the key effectors responsible for the characteristic morphological aspects of apoptosis, i.e. rounding up of the cell, reduction of cellular and nuclear volume, nuclear fragmentation, plasma membrane swelling etc. [6]. Caulin et al. observed that CK18 is cleaved by caspase- $6,-3$ and -7 , resulting in the appearance of a specific neo-epitope, which can be measured using an enzyme-linked immunosorbent assay (ELISA) [5].

A number of studies already investigated the value of total and caspase-cleaved CK18 as tumor marker in gastro-intestinal[7], breast[8] and testicular[9] cancer and showed that the measurement of ccCK18, as a marker of apoptosis, and uncleaved CK18, released during both apoptosis and necrosis, allows to distinguish between different cell death modes [10].

In this work, we studied the correlation between ccCK18 levels and PTX treatment efficacy in vitro and in vivo. Pharmacodynamic modelling was used to characterize the longitudinal changes in cell count and released ccCK18 levels from the in vitro experiments. 
III. Methods

\section{IN VITRO STUDY}

a. Cell lines and culture conditions

The human ovarian cancer cell lines PA-1, Caov-3 and SK-OV-3 were obtained from ATCC (LGC Standards S.a.r.I., France). SK-OV-3LucIP1 cells were selected in vivo [11] and together with the NIH:OVCAR-3 cell line provided by the LECR research group (Ghent University, Belgium). This cell panel was extended with the patient-derived xenograft-derived low-grade serous ovarian cancer cell line PM-LGSOC-01[12]. SK-OV-3 cells were maintained in ATCC-formulated McCoys 5A medium, SK-OV-3LucIP1 and Caov-3 cells in ATCCformulated Dulbecco's Modified Eagle's Medium (DMEM) and PA-1 together with PM-LGSOC-01 cells in ATCC-formulated Eagle's Minimum Essential medium (EMEM). NIH:OVCAR-3 cells were maintained in RPMI1640 Medium (Life Technologies, Belgium). All growth media were completed by adding $10 \%$ fetal bovine serum (FBS) and antibiotics (penicillin/streptomycin), and incubated at $37^{\circ} \mathrm{C}$ with $5 \% \mathrm{CO}_{2}$ in air.

b. Drug treatments

Cells were suspended in culture medium and added to a 96 -well plate in a volume of $200 \mu \mathrm{L}$ with a concentration of 6000 cells. Cells were allowed to attach for 72 hours before treating them with paclitaxel (PTX, Enzo Life Sciences BVBA, Belgium). A 1\% EtOH-solution in complete growth medium was used as control solution ( $0 \mathrm{nM}$ ) and vehicle for PTX-containing solutions. PTX concentrations in the clinically relevant concentration range of 1 to $1000 \mathrm{nM}[13]$ were studied. After incubating the cells with $0,1,10,100$ or 1000 nM PTX for 24 hours, wells were washed with phosphate buffered saline and complete growth medium before adding $200 \mu \mathrm{L}$ of fresh complete growth medium. Every $24 \mathrm{~h}$, up to $120 \mathrm{~h}$ after PTX treatment, medium was collected, levels of released ccCK18 biomarker were measured and an MTS assay was performed. For every concentration and time point, the experiment was performed in fourfold.

c. MTS assay

The MTS assay was performed according to the manufacturer's instructions, using the CellTiter $96^{\circ}$ AQueous 
Infinite $\oplus^{\bullet}$ M200 PRO NanoQuant plate reader (TECAN, Switzerland). Cell counts were assessed by performing MTS assays in the presence of standard curves using increasing cell amounts.

The cell culture medium aspirated from the wells was first centrifuged for $5 \mathrm{~min}$ at $1000 \mathrm{rpm}$ and then aliquoted and stored at $-80^{\circ} \mathrm{C}$ until analysis. In order to be certain that only cell-free supernatant was collected, an examination under the AE2000 binocular microscope (Motic Instruments Inc., Canada) was performed. Levels of ccCK18 were quantitatively determined, according to the manufacturer's instructions, using the M30 Apoptosense ${ }^{\circ}$ ELISA kit (PEVIVA, Sweden).

e. Cell lysates and Western blot analysis

Proteins were extracted from untreated cancer cells using the Laemmli lysis buffer $(0.125 \mathrm{M}$ Tris- $\mathrm{HCl}, 10 \%$ glycerol, $2.3 \%$ sodium dodecyl sulfate (SDS), $\mathrm{pH} 6.8$ ). Cell lysates were suspended in reducing sample buffer (1 M Tris- $\mathrm{HCl}, 30 \%$ glycerol, 6\% SDS, 3\% $\beta$-mercaptoethanol, 0.005\% bromophenol blue, $\mathrm{pH} 6.8$ ) and boiled for 5 minutes at $95^{\circ} \mathrm{C} .25 \mu \mathrm{g}$ proteins of each cell line were exposed to a 10\% SDS-PAGE gel and transferred to nitrocellulose membranes (Bio-Rad, Hercules, CA, USA). After blocking the membranes using $5 \%$ non-fat milk in phosphate-buffered saline (PBS) with 0.5\% Tween ${ }^{\circledR} 20$ (Sigma-Aldrich, Belgium), the blot was incubated overnight at $4^{\circ} \mathrm{C}$ with mouse monoclonal anti-human cytokeratin 18 (R\&D Systems, United Kingdom). After washing the membrane, incubation with HRP-conjugated anti-mouse IgG secondary antibody was performed at room temperature for 1 hour. The WesternBright Quantum HRP substrate (Advansta, Menlo Park, CA, USA) was added to the membranes to capture the luminescent signal using the Proxima 2850 Imager (IsoGen Life Sciences, De Meern, The Netherlands). Equal loading of samples was verified by primary antibodies mouse monoclonal anti-GAPDH (clone GAPDH-71.1, Sigma-Aldrich, Belgium).

f. Phase-nonspecific pharmacodynamic model for cell count

128 Cell count data were analyzed using a phase-nonspecific pharmacodynamic model with NONMEM ${ }^{\circledR}$ (version 129 7.3.0, ICON, Hanover, MD, USA) based on the work by Mouton et al. (1997)[14], Nielsen et al. (2007)[15] and 130 Rao et al. (2016) [16]. 


$$
\frac{d N}{d t}=\left(k_{n g}-\frac{E m a x \times \operatorname{Conc}}{C_{50}+\operatorname{Conc}}\right) \times N
$$

132 where $N$ refers to the cell number, $t$ is time, $k_{n g}$ is the net growth rate constant and $E_{\max }$ refers to the maximum PTX-induced treatment effect. Conc refers to the concentration of PTX to which cells were exposed and $C_{50}$ denotes the concentration at which half of the maximum effect is reached.

135 There was also a term base_count included in this model to take into account the estimated cell numbers 136 present before treatment with PTX. Population parameters and error variance were estimated using the 137 first-order (FO) estimation routine.

The ccCK18 release data were also analyzed using a phase-nonspecific pharmacodynamic model with NONMEM $^{\circledR}$ (version 7.3.0, ICON, Hanover, MD, USA). The term $\mathrm{k}_{\text {prod }}$ was used here as the net production rate constant of extracellular ccCK18. Parameters such as the maximum amount of released marker $\left(A_{\max }\right)$ and the Hill coefficient $\gamma$, to describe the steepness of the relationship between PTX concentration and response, were added. $A$ refers here to the extracellular amount of ccCK18.

$$
\frac{d A}{d t}=\left\{k_{\text {prod }} \times\left(1-\frac{A}{A \max }\right)+\frac{E \max \times \operatorname{Conc}^{\gamma}}{C_{50}^{\gamma}+\operatorname{Conc}^{\gamma}}\right\} \times A
$$

Also here the base_amount term was included in the model to estimate the initial amount of released ccCK18 before exposure to PTX. Population parameters and error variance were estimated using the first$\operatorname{order}(\mathrm{FO})$ method.

2. IN VIVO STUDY

a. PA-1 and SK-OV-3LuclP1 xenograft models

150 The human ovarian cancer cell lines PA-1 and SK-OV-3LucIP1 cells were used to xenograft SCID/Beige mice 151 (mice were commercially obtained from Envigo, The Netherlands). SK-OV-3LucIP1 cells were cultured in ATCC-formulated Dulbecco's Modified Eagle's Medium (DMEM) and PA-1 cells in ATCC-formulated Eagle's 
154 (FBS) and antibiotics (penicillin/streptomycin). Cells were incubated at $37^{\circ} \mathrm{C}$ with $5 \% \mathrm{CO}_{2}$ in air. 7 to 9 -week155 old female SCID/Beige mice were unilaterally subperitoneally injected with $1 \times 10^{6}$ cancer cells (1:1 serum free 156 medium:Matrigel (Corning, The Netherlands). In total, 20 PA-1-xenografted mice and 30 SK-OV-3LuclP1157 xenografted mice where studied. The control groups were based on 10 PA-1- and 15 SK-OV-3LuclP1xenografted mice, respectively. The maximum caging density was 6 from the same experimental group. All mice were maintained on a regular diurnal lighting cycle (12:12 light:dark) with ad libitum access to food and water. Mice were monitored once every day.

b. Single dose study

Mice were randomly assigned to the placebo or treatment group after subperitoneal tumor cell injection. PTX (Abraxane ${ }^{\circledR}$, Celgene, US) was intraperitoneally (ip, $50 \mathrm{mg} / \mathrm{kg}$ ) administered to the treatment group 2.5 weeks after tumor cell injection whereas the control group received an intraperitoneal injection of $0.9 \%$ $\mathrm{NaCl}$ (placebo) in an equal volume at the same time. Whole blood was collected via cardial puncture in K3EDTA-treated Sarstedt (B.V.B.A. Berchem, Belgium) Microvette ${ }^{\circledR} 200$ tubes, prior to obtain plasma. Samples were collected up to 2 weeks after PTX administration to measure ccCK18 levels. Blood collection via cardial puncture was considered a terminal procedure before mice were euthanized using an isoflurane overdose prior to cervical dislocation. Every individual mouse was considered an experimental unit in this study. The animals were excluded from the study if no tumor was present 2.5 weeks after tumor cell 171 injection, which was not the case here.

173 Tumor volume in xenografted mice was assessed using ultrasound imaging. Transparent ultrasound 174 transmission Polaris II gel (Ondes \& Rayons Medical, France) was applied to bare skin and a MicroScan ${ }^{\mathrm{TM}}$ MS550D (22-55 MHz, VisualSonics Inc., Canada) transducer with the Vevo ${ }^{\circledR} 2100$ imaging system (VisualSonics 176 Inc., Canada) was used to analyze the tumor volume in Vevo LAB 1.7.1 (VisualSonics Inc., Canada). Tumor 177 volumes (in $\mathrm{mm}^{3}$ ) were measured before receiving the single dose of PTX and before the cardial puncture at 178 the time of sacrifice. 
Tumor tissues collected from mice were fixed overnight in neutral buffered $10 \%$ formalin solution (SigmaAldrich, Belgium) and processed in the tissue core facility at Ghent University Hospital.

Collected plasma samples were aliquoted and stored at $-80^{\circ} \mathrm{C}$ until analysis. Levels of ccCK18 were quantitatively determined, according to the manufacturer's instructions, using the M30 Apoptosense ELISA kit (PEVIVA, Sweden).

IV. Results

Compared to the control solution, PTX concentrations of 1 to $1000 \mathrm{nM}$ were found to affect cell counts over time for all tested cell lines. Table 1 shows the estimated parameters, their relative standard errors (RSE) and 95\% confidence intervals (Cls) for the phase-nonspecific models on cell count.

All timepoints, up to $120 \mathrm{~h}$ post treatment, were taken into account in this model for all cell lines, except for

SK-OV-3. As for the SK-OV-3 profiles over time only a clear difference between PTX concentrations was noticed starting $96 \mathrm{~h}$ post treatment, only the last two timepoints were included in the model.

From Table 1 we see that all parameters are estimated with good precision except for $\mathrm{C}_{50}$ in SK-OV-3 and SK-

OV-3luclP1 cells as the large confidence intervals (Cls) are indicative for a high level of uncertainty. The estimated $\mathrm{C}_{50}$ S for SK-OV-3luclP1 and SK-OV-3 were $85.2 \mathrm{nM}$ and $6080 \mathrm{nM}$, respectively, suggesting that SKOV-3luclP1 is less sensitive compared to the other studied cell lines but with an estimated $C_{50}$ within the tested concentration range whereas SK-OV-3 is rather insensitive to PTX with an estimated $\mathrm{C}_{50}$ outside the tested concentration range. To avoid difficulties with the estimation fitted, a net growth rate parameter was included for each cell line instead of separate rate constants for natural cell growth and cell death. The 
$\mathrm{h}^{-1}$ ). For SK-OV-3, we were not able to separately estimate $E_{\max }$ and $\mathrm{C}_{50}$ due to the limitations of the data,

hence $E_{\max }$ was fixed to the mean $E_{\max }$ of all five other cell lines. The estimated $E_{\max }$ parameter was lowest in

PM-LGSOC-01 $\left(0.0151 \mathrm{~h}^{-1}\right)$ compared to similar estimates of about $0.025 \mathrm{~h}^{-1}$ for the other cell types. We also

found that the estimated base_count parameter, representing the amount of cells that were present prior to

exposure to PTX, was different across cell lines. Based on these estimates, cell division and growth is considered being slower in SK-OV-3lucIP1 in contrast to the other cell lines with PM-LGSOC-01 having the highest amount of cells at the start of the therapy.

b. Western blotting

Figure 1 illustrates the differences in CK18 expression at PTX-free cell level. These results show that expression of CK18 is lower in SK-OV-3LucIP1 compared to the other cell lines.

c. In vitro release of ccCK18

214 The estimated values for the fitted phase-nonspecific pharmacodynamic model on released ccCK18 levels are presented in Table 2, accompanied by the percent relative standard error (RSE) and 95\% confidence intervals (Cls) for all parameters. This table illustrates a slower release rate for ccCK18 (Krod) in PM-LGSOC$01\left(0.0155 \mathrm{~h}^{-1}\right)$ and PA-1 $\left(0.0177 \mathrm{~h}^{-1}\right)$ cells compared to the other cell lines. The estimated $E_{\max }$, the effect of difference in $\mathrm{C}_{50}$ value was observed between NIH:OVCAR-3 $(8.8 \mathrm{nM})$ and Caov-3 $(0.796 \mathrm{nM})$ cells. A Hill coefficient of 8.02 was estimated for PM-LGSOC-01 compared to 2.21 for SK-OV-3, indicating a steeper drug concentration-effect relationship in PM-LGSOC-01 compared to SK-OV-3. Based on the base_amount and $\mathrm{A}_{\max }$ parameters, SK-OV-3lucIP1 and SK-OV-3 had the lowest initial extracellular ccCK18 levels and also the lowest levels of maximum releasable amounts of ccCK18. For SK-OV-3lucIP1, the lower estimated $A_{\max }$ value of 907 was also in line with the western blot result as expression of CK18 was demonstrated to be lower compared to the other cell types. 
228 Figure 2 illustrates the relationship between cell count and released ccCK18 levels. Here, the model 229 predictions for both cell count and released levels of ccCK18 are plotted over time. The calculated 230 Spearman's rank correlation coefficient was highest for SK-OV-3lucIP1 (0.89, 95\% Cl: 0.75-0.95), followed by PA-1 (0.87, 95\% Cl: 0.71-0.94), NIH:OVCAR-3 (0.76, 95\% Cl: 0.51-0.89), PM-LGSOC-01 (0.73, 95\% Cl: 0.450.87), SK-OV-3 (0.71) and Caov-3 (0.64, 95\% Cl: 0.32-0.83). Changes in cell count are associated with proportionally larger increases in ccCK18 for PM-LGSOC-01 and SK-OV-3. This is seen from Figure 2 where most data points lie above the identity line. In contrast, reductions in cell count, at higher PTX levels, are accompanied with less than proportional increases in ccCK18 for NIH:OVCAR-3. As observed from Figure 2, at later timepoints, ccCK18 levels start decreasing or reach a plateau.

a. Relationship between tumor volume and released ccCK18 levels

Due to the observed differences in sensitivity to PTX and ccCK18 release in vitro, xenografts based on PA-1 and SK-OV-3lucIP1 cells were selected to be studied in vivo. Figure 3 illustrates the in vivo observed relationship between tumor volume and released ccCK18 levels. Regarding tumor volume, it is interesting to see that it takes about 1 week before a drop in tumor volume was observed in the PA-1 xenografts whereas no clear drop in tumor volume was observed for the SK-OV-3lucIP1 xenografts. When looking at the released ccCK18 levels, SK-OV-3lucIP1 tumors present with more similar released ccCK18 levels over time between control and treatment group whereas the PTX-treated PA-1 tumors clearly show an initial peak of ccCK18 after PTX treatment before normalizing after about 1 week.

b. Immunohistochemistry

248 From both the control and $50 \mathrm{mg} / \mathrm{kg}$ intraperitoneally treated PA-1 and SK-OV-3luclP1 groups, tissue 249 samples were stained for H\&E, proliferation marker Ki67 and CK8-18 marker. The latter was only studied in the control groups. In addition, it is important to note that both cancer cell lines do represent a very 

germ cell tumor PA-1). In Figure 4, expression of Ki67 confirmed the ongoing tumor cell proliferation in both cell line-derived xenografts post therapy. Expression of CK8-18 in the control group illustrated the basis for later potential caspase cleavage caused by PTX.

V. Discussion

257 As many chemotherapeutic agents cause apoptosis, the potential of ccCK18 as a quantitative pharmacodynamic biomarker and hence, a helpful decision tool in treatment optimization was evaluated here. In this study, next to observed differences in sensitivity to PTX, the correlation between cell count and apoptotic cell death marker ccCK18 was shown in ovarian cancer cell lines SK-OV-3lucIP1, PA-1, NIH:OVCAR3, PM-LGSOC-01, SK-OV-3 and Caov-3. Based on the in vitro results, SK-OV-3lucIP1 and PA-1 xenografts were studied in vivo representing, respectively, a model less sensitive and sensitive to PTX. In vivo, a correlation between released ccCK18 levels and tumor volume was observed in PA-1 xenografts whereas no clear effect of PTX was found on tumor volume or ccCK18 levels over time in the SK-OV-3lucIP1 xenografted mice.

265 The $\mathrm{C}_{50}$ values observed, based on the in vitro model, are hard to compare with previously published work due to differences in experimental conditions (such as drug concentration and exposure time). Compared to our $C_{50}$ of about $6000 \mathrm{nM}$ for the SK-OV-3 cells, Au et al. [17] found a $C_{50}$ equal to $5 \mathrm{nM}$ for SK-OV-3 exposing the cells $24 \mathrm{~h}$ to PTX and measuring the drug effect after $96 \mathrm{~h}$. Possible reasons for the variability in observed $\mathrm{C}_{50}$ value for SK-OV-3 in both studies could be related to cell cycle, confluency and/or passage effects [18]. It is also important to highlight that in this experiment $C_{50}$ was estimated considering a 1 week period whereas commonly only one single timepoint is used. In addition, the difference in $\mathrm{C}_{50}$ values reported between cell types can also be explained by heterogeneous responses to anticancer treatments [19].

273 Brandt and colleagues (2010)[7] concluded that circulating levels of ccCK18 might be a useful biomarker to monitor treatment response in patients with gastrointestinal cancers. A correlation between intact and 
caspase-cleaved forms of CK18 levels and clinical response to therapy in breast cancer was observed by observed changes in total and caspase-cleaved CK18 during chemotherapy[9]. As a result, measuring soluble keratin protein fragments in the clinic is believed to be of great value for the early detection of tumor progression, metastasis formation and for a quick evaluation of the therapeutic response in epithelial malignancies [20]. Outside the field of oncology, serum CK18 levels have also been studied as cell death markers in (alcoholic) hepatitis[21, 22] and in patients with cirrhosis[23] or drug-induced liver injury[24] . In our in vitro study, at later time points, deviating biomarker release patterns were observed which can be explained by the loss of M30 reactivity as reported in apoptotic cells[25] or by the different ongoing processes during cell death[26]. As PTX induces cell death processes, Lieu at al. [27] described the apoptogenic mechanisms after PTX treatment to be mitotic arrest and microtubule damage, the first one induced at PTX concentrations below 200 nM and the last one at higher PTX concentrations. As a result, high concentrations of PTX are able to induce apoptosis independently of mitotic arrest at any phase of the cell cycle.

Next to differences in CK18 expression at the cell level[28], also variation in population doubling time between these different cell lines may explain our observed differences in ccCK18 release post therapy. As a result, a higher number of cell divisions will be accompanied by a longer duration of drug present to a larger number of cells. Next to an extended drug effect, the previously mentioned phenomenon might also cause an increase in the specific cell fraction subjected to PTX toxicity [17]. In addition, the cell fraction passing through the mitotic phase will also play a crucial role with regard to the release of ccCK18, since PTX-induced cell cycle arrest and apoptosis are mainly occurring in the mitotic phase [29]. We did not discriminate between the G1, S, G2 and M phase in this study in accordance with the clinical setting in which equally no cellular analysis of the complete tumor is possible before treating the patient. 
agreement with the early-stage apoptosis reflected by the M30 ELISA as M30 does not measure late apoptosis stages including generation of secondary CK18 caspase cleavage products[30]. Induction of apoptosis by chemotherapeutic agents is typically characterized by slow kinetics over 24 hours. Regarding paclitaxel, as this compound is retained in tumors for over 5 days [31], CK18 is typically cleaved after more than 12 hours of drug exposure [32]. Levels of ccCK18 are likely a better marker to inform on PTX-induced tumor cell death for the PA-1 group compared to the SK-OV-3lucIP1 group. Nevertheless, the small number of animals used did not allow to draw firm conclusions on the potential of ccCK18 to provide significant information regarding chemotherapeutic responses.

308 Given the complexity of a disease like ovarian cancer and the variety of cell death processes, we believe another potential explanation for our in vivo findings, i.e. to consider ccCK18 a better marker for effects of PTX on PA-1 cells compared to SK-OV-3luclP1 cells, might be related to the involvement of Akt[33]. The serine-threonine kinase Akt is known to exert anti-apoptotic effects through several downstream targets. It is also known that Akt is cleaved during mitochondrial-mediated apoptosis in a caspase-dependent manner. The observation that cleavage of Akt occurs during apoptosis suggests that either a level of baseline Akt signaling is vital for cell survival or that Akt activation occurs during apoptosis and acts as a 'brake' on this process [34]. Since it is known that Akt inactivation sensitizes human ovarian cancer cells to paclitaxel, Kim and colleagues (2007)[33] explored the difference in Akt phosphorylation levels between PTX-sensitive PA-1 cells and PTX-resistant SK-OV-3 cells. In their study, a higher level of phosphorylated Akt in SK-OV-3 cells compared to PA-1 cells was shown. As a result, the link between Akt activity, PTX resistance and paclitaxelinduced apoptosis was hypothesized[35]. Our in vivo findings are also in line with this as we concluded that SK-OV-3lucIP1 is associated with less ccCK18 release as a result of less paclitaxel-induced caspase cleavage of CK18 compared to PA-1. Taking into account the higher Akt activity in SK-OV-3 cells observed by Kim et al. (2007)[33], mediation of survival signals by Akt might preserve SK-OV-3luclP1 cells from apoptotic pathways.

VI. Conclusion 
In conclusion, our in vitro study showed evidence of an association between PTX-induced cell toxicity and released ccCK18 levels. Based on our in vivo study with PTX-sensitive xenografts, we found that response to PTX was immediately shown as a peak in released ccCK18 whereas an observed decrease in tumor volume was rather delayed. Overall, these experiments indicate the potential of ccCK18 to inform on drug-induced cell death which can be informative to optimize cancer treatment.

VII. List of abbreviations

CK18 : cytokeratin 18

ccCK18: caspase-cleaved cytokeratin 18

PTX: paclitaxel

CK8: cytokeratin 8

ELISA: enzyme-linked immunosorbent assay

ATCC: American Type Culture Collection

DMEM: Dulbecco's Modified Eagle's Medium

EMEM: Eagle's Minimum Essential medium

FBS: fetal bovine serum

$\mathrm{Cl}$ : confidence interval

RSE: relative standard error

Kng: net growth rate constant

Emax: maximum effect

C50: concentration at $50 \%$ effect

Kprod: net production rate constant
VIII. Declarations

a. Ethics approval and consent to participate 
Animal experiments were conducted in accordance with the local ethics committee (ECD 16/11, Ghent

University Hospital). The reported study does not involve human participants, human data or human tissue.

b. Consent for publication

353 Not applicable as this manuscript does not contain data from any individual person.

c. Availability of data and material

355 The datasets used and/or analysed during the current study are available from the corresponding author on 356 reasonable request.

d. Competing interests

358 AV is an employee of Johnson \& Johnson and holds stock/stock options in the company. All other authors 359 declare no conflict of interest.

e. Funding

The authors are indebted to the Research Foundation Flanders (FWO) for financial support (research project

362 G016915N). This research funding was used to gather all the data and perform all mentioned analyses on the 363 collected samples.

f. Authors' contributions

365 EDT acquired and analyzed in vitro and in vivo data, wrote the manuscript. KVdV and JVD performed 366 pathological studies. AV and PC participated in data analysis. JVB participated in coordination of the project. 367 Critical revision of the manuscript and approval of the final version: all authors.

g. Acknowledgements

The authors thank the Laboratory of Toxicology (Faculty of Pharmaceutical Sciences, Ghent University, Belgium) and the Laboratory of Experimental Cancer Research (LECR - Faculty of Medicine and Health Care, 
374 1. M. B. Omary, et al., Keratin modifications and solubility properties in epithelial cells and in vitro.

2. E. Fuchs and D.W. Cleveland, A structural scaffolding of intermediate filaments in health and disease. Science, 1998. 279(5350): p. 514-519.

3. H. Kumemura, et al., Mutation in keratin 18 induces mitochondrial fragmentation in liver-derived epithelial cells. Biochemical and Biophysical Research Communications, 2008. 367(1): p. 33-40.

4. R. Moll, Cytokeratins as markers of differentiation in the diagnosis of epithelial tumors. Subcell Biochem, 1998. 31: p. 205-62.

5. C. Caulin, G.S. Salvesen, and R.G. Oshima, Caspase cleavage of keratin 18 and reorganization of intermediate filaments during epithelial cell apoptosis. Journal of Cell Biology, 1997. 138(6): p. 13791394.

6. Nancy A. Thornberry and Y. Lazebnik, Caspases: Enemies Within. Science, 1998. 281(5381): p. 13121316.

7. Brandt, D., et al., Serum biomarkers of cell death for monitoring therapy response of gastrointestinal carcinomas. European Journal of Cancer, 2010. 46(8): p. 1464-1473.

8. Olofsson, M.H., et al., Cytokeratin-18 Is a Useful Serum Biomarker for Early Determination of Response of Breast Carcinomas to Chemotherapy. Clinical Cancer Research, 2007. 13(11): p. 3198.

9. de Haas, E.C., et al., Clinical Evaluation of M30 and M65 ELISA Cell Death Assays as Circulating Biomarkers in a Drug-Sensitive Tumor, Testicular Cancer. Neoplasia, 2008. 10(10): p. 1041-1048.

10. S. Linder, et al., Determining tumor apoptosis and necrosis in patient serum using cytokeratin 18 as a biomarker. Cancer Letters, 2004. 214(1): p. 1-9.

11. Elly De Vlieghere, et al., Data on in vivo selection of SK-OV-3 Luc ovarian cancer cells and intraperitoneal tumor formation with low inoculation numbers. Data in Brief, 2016. 6: p. 542-549.

12. De Thaye, E., et al., Establishment and characterization of a cell line and patient-derived xenograft (PDX) from peritoneal metastasis of low-grade serous ovarian carcinoma. Scientific Reports, 2020. 10(1): p. 6688.

13. Jang, S.H., M.G. Wientjes, and J.L.S. Au, Determinants of Paclitaxel Uptake, Accumulation and Retention in Solid Tumors. Investigational New Drugs, 2001. 19(2): p. 113-123.

14. Mouton, J.W., A.A. Vinks, and N.C. Punt, Pharmacokinetic-pharmacodynamic modeling of activity of ceftazidime during continuous and intermittent infusion. Antimicrobial agents and chemotherapy, 1997. 41(4): p. 733-738.

15. Nielsen, E.I., et al., Semimechanistic pharmacokinetic/pharmacodynamic model for assessment of activity of antibacterial agents from time-kill curve experiments. Antimicrobial agents and chemotherapy, 2007. 51(1): p. 128-136.

16. Rao, G.G., et al., Translational Modeling of Antibacterial Agents, in Systems Pharmacology and Pharmacodynamics, D.E. Mager and H.H.C. Kimko, Editors. 2016, Springer International Publishing: Cham. p. 371-402.

17. J. L. S. Au, et al., Pharmacodynamics of immediate and delayed effects of paclitaxel: Role of slow apoptosis and intracellular drug retention. Cancer Research, 1998. 58(10): p. 2141-2148.

18. Wang Jin, et al., A computational modelling framework to quantify the effects of passaging cell lines. PLOS ONE, 2017. 12(7): p. e0181941.

19. Benjamin Haibe-Kains, et al., Inconsistency in large pharmacogenomic studies. Nature, 2013. 504(7480): p. 389-393.

20. V. Barak, et al., Clinical utility of cytokeratins as tumor markers. Clinical Biochemistry, 2004. 37(7): p. 529-540.

21. Parfieniuk-Kowerda, A., et al., Serum cytochrome $c$ and m30-neoepitope of cytokeratin-18 in chronic hepatitis C. Liver International, 2014. 34(4): p. 544-550.

22. Bissonnette, J., et al., A Prospective Study of the Utility of Plasma Biomarkers to Diagnose Alcoholic Hepatitis. Hepatology, 2017. 66(2): p. 555-563. 
23. Macdonald, S., et al., Cell death markers in patients with cirrhosis and acute decompensation. Hepatology, 2018. 67(3): p. 989-1002.

24. Church, R.J., et al., Candidate biomarkers for the diagnosis and prognosis of drug-induced liver injury: An international collaborative effort. Hepatology, 2019. 69(2): p. 760-773.

25. Tao, G.Z., et al., Monitoring of epithelial cell caspase activation via detection of durable keratin fragment formation. The Journal of pathology, 2008. 215(2): p. 164-74.

26. Yeung, T.K., et al., The Mode of Action of Taxol: Apoptosis at Low Concentration and Necrosis at High Concentration. Biochemical and Biophysical Research Communications, 1999. 263(2): p. 398-404.

27. C. H. Lieu, Y. N. Chang, and Y.K. Lai, Dual cytotoxic mechanisms of submicromolar taxol on human leukemia HL-60 cells. Biochemical Pharmacology, 1997. 53(11): p. 1587-1596.

28. Stimpfl, M., et al., Expression of mucins and cytokeratins in ovarian cancer cell lines. Cancer letters, 1999. 145(1-2): p. 133-41.

29. M.A. Jordan, et al., Mechanism of mitotic block and inhibition of cell-proliferation by taxol at low concentrations. Proceedings of the National Academy of Sciences of the United States of America, 1993. 90(20): p. 9552-9556.

30. Ku, N.-O., et al., Keratins: Biomarkers and modulators of apoptotic and necrotic cell death in the liver. Hepatology, 2016. 64(3): p. 966-976.

31. Mori, T., et al., Retention of paclitaxel in cancer cells for 1 week in vivo and in vitro. Cancer chemotherapy and pharmacology, 2006. 58(5): p. 665-72.

32. Gerhard, H., Cytokeratin 18 (CK18) and Caspase-Cleaved CK18 (ccCK18) as Response Markers in Anticancer Therapy, Cytokeratins - Tools in Oncology IntechOpen, 2012.

33. Kim, S.-H., Y.-S. Juhnn, and Y.-S. Song, Akt Involvement in Paclitaxel Chemoresistance of Human Ovarian Cancer Cells. Annals of the New York Academy of Sciences, 2007. 1095(1): p. 82-89.

34. Tang, D., et al., Akt is activated in response to an apoptotic signal. Journal of Biological Chemistry, 2001. 276(32): p. 30461-30466.

35. Page, C., et al., Overexpression of Akt/AKT can modulate chemotherapy-induced apoptosis. Anticancer research, 2000. 20: p. 407-16.

\section{Table and Figure legends}

\section{Table 1. Phase-nonspecific model parameters on cell count}

\section{Table 2. Phase-nonspecific model parameters on released ccCK18}

Figure 1. Western blot of CK18 expression. Western blot to evaluate expression of CK18 at cell level. GAPDH was used as loading control. The original blot is presented in Supplementary Figure S1.

Figure 2. In vitro relationship between cell count and released ccCK18 levels. Relationship between cell count and released ccCK18 levels. These graphs are based on the predicted data from the different phasenonspecific pharmacodynamic models. On the $\mathrm{x}$-axis cell count is represented as the following ratio: prediction for control (0 nM) over prediction for a specific PTX level, M30 on the $y$-axis represents ccCK18 using the ratio calculated from prediction for a specific PTX level over prediction for control (0 nM). The grey 
461 line in all graphs illustrates the line of identity. From left to right, the symbols are directly related to the 462 specified time points studied (0, 24, 48, 72, 96, 120 and 144h). This figure was made using GraphPad Prism 8 463 (GraphPad Software, Inc., USA).

464 Figure 3. In vivo relationship between tumor volume and released ccCK18 levels. Relationship between 465 tumor volume and released ccCK18 levels. The left panels represent data from PA-1 xenografts whereas the 466 right panels illustrate the SK-OV-3lucIP1 xenografts. These graphs are based on measured tumor volume 467 ( $\mathrm{mm}^{3}$, upper panel) and measured ccCK18 levels (pM, lower panel) using the M30 Apoptosense ${ }^{\circledR}$ Elisa 468 (PEVIVA, Sweden). Grey triangles and lines represent the PTX-treated group of cell line-derived xenografts 469 whereas the black triangles and lines are illustrating the non-PTX-treated control groups.

470 Figure 4. Immunostaining of tumor tissue. Tumor tissue samples stained for H\&E, proliferation marker Ki67 471 and CK8-18 marker (only control group). Scale bars represent $1 \mathrm{~mm}$, except for the PTX-treated SK-OV-

472 3luclP1 group where the scale bar represents $200 \mu \mathrm{m}$.

473 Supplementary Figure S1. Original western blot. Original western blot of Figure 1 as Figure 1 is the result of 474 the overlay of the images presented in this Supplementary Figure. 
Table 1.

\begin{tabular}{|c|c|c|c|c|c|c|}
\hline & $\begin{array}{c}\text { PM-LGSOC- } \\
01\end{array}$ & Caov-3 & NIH:OVCAR-3 & PA-1 & $\begin{array}{l}\text { SK-OV- } \\
\text { 3lucIP1 }\end{array}$ & SK-OV-3 \\
\hline $\begin{array}{l}\text { Kng }\left(h^{-1}\right) \\
(\text { RSE\%) } \\
(95 \% \mathrm{Cl})\end{array}$ & $\begin{array}{c}0.0077 \\
(8 \%) \\
(0.007-0.009)\end{array}$ & $\begin{array}{c}0.0038 \\
(20 \%) \\
(0.002-0.005)\end{array}$ & $\begin{array}{c}0.0089 \\
(7 \%) \\
(0.008-0.01)\end{array}$ & $\begin{array}{c}0.0055 \\
(10 \%) \\
(0.004-0.007)\end{array}$ & $\begin{array}{c}0.0164 \\
(7 \%) \\
(0.014-0.019)\end{array}$ & $\begin{array}{c}0.0036 \\
(27 \%) \\
(0.002-0.005)\end{array}$ \\
\hline $\begin{array}{c}\text { Emax }\left(h^{-1}\right) \\
(\text { RSE\%) } \\
(95 \% \mathrm{Cl})\end{array}$ & $\begin{array}{c}0.0151 \\
(4 \%) \\
(0.014-0.016)\end{array}$ & $\begin{array}{c}0.0236 \\
(6 \%) \\
(0.021-0.026)\end{array}$ & $\begin{array}{c}0.0299 \\
(3 \%) \\
(0.028-0.031)\end{array}$ & $\begin{array}{c}0.0288 \\
(3 \%) \\
(0.027-0.031)\end{array}$ & $\begin{array}{c}0.0253 \\
(14 \%) \\
(0.018-0.032)\end{array}$ & 0.0245 FIX \\
\hline $\begin{array}{l}\text { C50 (nM) } \\
\text { (RSE\%) } \\
(95 \% \mathrm{Cl})\end{array}$ & $\begin{array}{c}4.02 \\
(14 \%) \\
(2.905-5.135)\end{array}$ & $\begin{array}{c}4.85 \\
(27 \%) \\
(2.263-7.437)\end{array}$ & $\begin{array}{c}7.24 \\
(15 \%) \\
(5.143-9.337)\end{array}$ & $\begin{array}{c}22.3 \\
(10 \%) \\
(17.733- \\
26.867)\end{array}$ & $\begin{array}{c}85.2 \\
(56 \%) \\
(-7.9-178.3)\end{array}$ & $\begin{array}{c}6080 \\
(55 \%) \\
(-505.6- \\
12665.6)\end{array}$ \\
\hline $\begin{array}{c}\text { Base_count } \\
\text { (RSE\%) } \\
(95 \% \mathrm{Cl})\end{array}$ & $\begin{array}{c}89322 \\
(0 \%) \\
(80018-99 \\
708)\end{array}$ & $\begin{array}{c}10615 \\
(1 \%) \\
(9653-11673)\end{array}$ & $\begin{array}{c}15522 \\
(0 \%) \\
(14705-16384)\end{array}$ & $\begin{array}{c}36316 \\
(0 \%) \\
(33423-39459)\end{array}$ & $\begin{array}{c}3905 \\
(1 \%) \\
(3492-4368)\end{array}$ & $\begin{array}{c}44356 \\
(1 \%) \\
(37161-52945)\end{array}$ \\
\hline
\end{tabular}

478

479

480 
Table 2.

\begin{tabular}{|c|c|c|c|c|c|c|}
\hline & PM-LGSOC-01 & Caov-3 & NIH:OVCAR-3 & PA-1 & SK-OV-3lucIP1 & SK-OV-3 \\
\hline Kprod $\left(h^{-1}\right)$ & 0.0155 & 0.0267 & 0.0333 & 0.0177 & 0.0228 & 0.0237 \\
\hline (RSE\%) & $(5 \%)$ & $(3 \%)$ & $(3 \%)$ & $(4 \%)$ & $(4 \%)$ & $(8 \%)$ \\
\hline$(95 \% \mathrm{Cl})$ & $(0.014-0.017)$ & $(0.025-0.028)$ & $(0.031-0.035)$ & $(0.016-0.019)$ & $(0.021-0.025)$ & $(0.02-0.027)$ \\
\hline $\operatorname{Emax}\left(\mathrm{h}^{-1}\right)$ & 0.0428 & 0.008 & 0.0087 & 0.02 & 0.0076 & 0.0128 \\
\hline (RSE\%) & (3\%) & (9\%) & (9\%) & $(3 \%)$ & $(5 \%)$ & (9\%) \\
\hline$(95 \% \mathrm{Cl})$ & $(0.041-0.045)$ & $(0.007-0.009)$ & $(0.007-0.01)$ & $(0.019-0.021)$ & $(0.007-0.008)$ & $(0.011-0.015)$ \\
\hline C50 (nM) & 8.13 & 0.796 & 8.8 & 1.49 & 7.37 & 4.53 \\
\hline (RSE\%) & $(2 \%)$ & $(3 \%)$ & $(6 \%)$ & $(3 \%)$ & $(5 \%)$ & $(28 \%)$ \\
\hline$(95 \% \mathrm{Cl})$ & $(7.752-8.508)$ & $(0.756-0.836)$ & (7.724-9.876) & $(1.407-1.573)$ & $(6.659-8.081)$ & $(2.06-7)$ \\
\hline Hill factor $\gamma$ & 8.02 & 5.46 & 6.71 & 5.31 & 6.58 & 2.21 \\
\hline (RSE\%) & $(8 \%)$ & $(16 \%)$ & $(24 \%)$ & $(5 \%)$ & $(3 \%)$ & $(30 \%)$ \\
\hline$(95 \% \mathrm{Cl})$ & $(6.83-9.21)$ & (3.751-7.169) & (3.515-9.905) & $(4.747-5.873)$ & (6.249-6.911) & $(0.928-3.492)$ \\
\hline Base_amount & 137 & 203 & 102 & 150 & 20.5 & 22.2 \\
\hline (pM) & $(2 \%)$ & $(1 \%)$ & $(1 \%)$ & $(1 \%)$ & $(2 \%)$ & $(4 \%)$ \\
\hline (RSE\%) & $(119-159)$ & $(185-222)$ & (91.4-113) & $(136-165)$ & $(17.8-23.5)$ & $(17.5-28.2)$ \\
\hline $\operatorname{Amax}(p M)$ & 2345 & 3395 & 2644 & 4024 & 907 & 838 \\
\hline (RSE\%) & $(1 \%)$ & $(1 \%)$ & $(1 \%)$ & $(1 \%)$ & $(2 \%)$ & $(3 \%)$ \\
\hline$(95 \% \mathrm{Cl})$ & (2 156-2 551) & (2 097- 3 722) & (2 390-2 925) & (3 419-4 737) & $(733-1$ 122) & (590-1 190) \\
\hline
\end{tabular}

